\title{
Hemoglobin Electrophoretic Patterns in Punjabi Children With
} Anemia

\author{
Kaur Puneet ${ }^{1,}$ Singh Daljit ${ }^{2}$ Kukreja Sahiba ${ }^{3}$, Manjari Mridu ${ }^{4}$ \\ ${ }^{1} M D$ - Pathology, Department Pathology \\ ${ }^{2} \mathrm{MD}-$ Pathology,Department Pathology \\ ${ }^{3} M D$ - Biochemistry, Department Biochemistry \\ ${ }^{4} \mathrm{MD}-$ Pathology, Department Pathology \\ Sri Guru Ram Das Institute Of Medical Sciences And Research, Amritsar, Punjab
}

\begin{abstract}
Context: Carrier frequency of hemoglobinopathy ranges from 3-17\% in Indian population. Early and accurate diagnosis of type of anemia is imperative and requires the use of hemoglobin electrophoresis.

Aims: To find the prevalence of hemoglobinopathy and different electrophoretic patterns in Punjabi population in subset of anemic children aged less than 18 years, by using agar gel hemoglobin electrophoresis method.

Methods and Material: A prospective study included 100 Punjabi children aged <18 years with hemoglobin $<9 g m \%$. Complete hemogram, red blood cell (RBC) indices and peripheral blood film (PBF) was examined. Hemoglobin electrophoresis was performed using agar gel electrophoresis method to find different electrophoretic patterns of hemoglobin in the study group.

Results: 100 cases were included with mean age (7.4 \pm .6years) and mean hemoglobin $(7.4 \pm 1.4 \mathrm{~g} / \mathrm{dl})$. Hemoglobin electrophoresis revealed abnormal hemoglobin electrophoretic pattern in five patients. Three patients had an abnormal $\mathrm{HbA}_{2}$ band (beta thalassemia trait); one patient had abnormal HbF and HbA band (beta thalassemia major); and one patient had an abnormal band in HbSD region (HbS confirmed by sickling). No statistically significant difference was noted in RBC indices, PBF examination in patients with normal versus abnormal hemoglobin pattern.

Conclusions: Blood parameters including RBC indices and PBF cannot help diagnose abnormal hemoglobin pattern. Hemoglobin electrophoresis by agar gel electrophoresis method is a cost effective method for screening patients with suspected hemoglobinopathies, however it is time consuming and requires proper standardization.
\end{abstract}

Keywords: Anemia, Hemoglobinopathy, Hemoglobin electrophoresis.

KeyMessages: Agar gel electrophoresis is a cost effective method to screen and diagnose hemoglobinopathy in a resource constraint country like India.

\section{Introduction}

The burden of hemoglobinopathies in India is tremendous with around 45 million total carriers and approximately 15,000 infants born each year with this gene defect. Carrier frequency of hemoglobinopathy ranges from 3-17\% in Indian population. It is imperative to make an early and accurate diagnosis of the type of anemia. Complete blood profile, red blood cell (RBC) indices and peripheral blood film (PBF) do not help to exclude hemoglobinopathy traits and thalassemia from nutritional causes of anemia. Hemoglobin electrophoresis at alkaline $\mathrm{pH}$ is the single most important investigation in preliminary screening of abnormal hemoglobin. In a developing country like India, where cost is a major concern, agar gel electrophoresis is an ideal technique for routine screening of abnormal hemoglobins. This study aims to find the prevalence of hemoglobinopathy and different electrophoretic patterns in Punjabi population in subset of anemic children aged less than 18 years, by using agar gel hemoglobin electrophoresis method.

\section{Subjects And Methods}

This was a prospective cohort study conducted in the department of Pathology, Sri Guru Ram Das Institute of Medical Sciences \& Research, Amritsar. It included 100 anemic children from Punjabi population aged less than 18 years with hemoglobin less than $9 \mathrm{~g} / \mathrm{dl}$. Using an automated cell counter, a complete hemogram including RBC indices was obtained and peripheral blood smear was examined in all cases.

Hemoglobin electrophoresis was then performed on cases using agar gel electrophoresis as per the following technique: ${ }^{[4]}$

$1.3 \mathrm{ml}$ of whole blood was obtained in an EDTA vials and refrigerated at $4^{\circ} \mathrm{C}$.

2. Hemolysate Preparation: Red cells washed thrice in excess of normal saline in a test tube. To the washed red cells sediments was added equal amount of distilled water mixed, thoroughly and then equal amount of 
solution of carbon tetrachloride was added. The contents were again thoroughly shaken to ensure complete red cell lysis. The lysate thus prepared was then processed.

3. Buffer Preparation: A Tris-EDTA-borate (TEB) buffer $\mathrm{pH} 8.6$ was used. It consists of the following

\section{Stock Buffer}

a. Tris (hydroxy methyl methylamine)

b. Ethylene Diamine Tetra-acetic acid

$109.00 \mathrm{gms}$

c. Boric acid

d. Distilled water upto

$1.85 \mathrm{gms}$

$30.92 \mathrm{gms}$

1 liter

\section{Working Buffer}

A 1:10 dilution of the stock buffer in distilled water was prepared for routine use.

4. Agar Gel Preparation: $500 \mathrm{mg}$ of pure E. Merck agar powder was dissolved in $50 \mathrm{ml}$ of working buffer to give a $1 \%$ agar gel. The contents were boiled till they turned uniformly transparent. $5 \mathrm{ml}$ of hot molten agar was poured over an evenly surfaced clean glass slide. The agar was allowed to cool and jellify at room temperature.

5. Charging Of Lysates: 3-5 $\mu \mathrm{g}$ of hemolysate was collected in thin bore Pasteur pipette and smeared over the edge of a cover slip approximately cut to give a width of $1 \mathrm{~cm}$. The smear edge was then charged briskly into the agar gel both for control and test. The contents were allowed to spread within the slit for a while and the cover slip was withdrawn by a brisk outward movement.

6. The charged plates were then placed in an electrophoretic chamber and the electrical circuit was completed by bringing the agar surface into contact with anodal and cathodal chambers of buffer solution through strips of filter paper. A current of $10 \mathrm{~mA}$ was passed for 60-90 minutes to give adequate separation of bands.

7. The slide thus run was fixed in pure methanol. The preparation was stained with $0.5 \%$ amido black solution (in Methanol) for 1-2 minutes. The excess of stain was washed out with 5\% acetic acid over a period of 30 minutes. The slide was then dried under hot air current.

8. The bands obtained from the patient's hemolysate were compared and matched with the control. Any abnormalities if present were then recorded.

9. According to the presence or lack of abnormalities seen in the band; all the patient samples of anemia were segregated as hemoglobinopathic samples and non hemoglobinopathic samples (nutritional or other causes of anemia).

All the cases with abnormal hemoglobinopathic samples were further sub categorized and analyzed.

\section{Results}

Total of 100 cases with male:female ratio of 1.6:1. The mean age distribution of the study population was $7.4 \pm 5.6$ years with mean age for male $6.3 \pm 5.2$ years and for female $9.1 \pm 5.7$ years. Mean hemoglobin in the study group was $7.4 \pm 1.4 \mathrm{~g} / \mathrm{dl}$. In 85 patients hemoglobin ranged between $6.1-8.9 \mathrm{~g} / \mathrm{dl}$, fourteen patients had hemoglobin between $3-6 \mathrm{~g} / \mathrm{dl}$ and only one case was below $3 \mathrm{~g} / \mathrm{dl}$. Mean haemoglobin value was $7.6 \pm 1.3 \mathrm{~g} / \mathrm{dl}$ for males and $7.2 \pm 1.6 \mathrm{~g} / \mathrm{dl}$ for females. (TABLE 1)

Red blood corpuscular (RBC) count was low in 82 patients in the study group, $31(81.6 \%)$ of female and $51(82.3 \%)$ of male patients had low RBC count. Eight patients had a RBC count more than normal. Low mean corpuscular volume (MCV) was seen in 74 patients, with a mean of $74.0 \pm 13.5 \mathrm{fL}$. A low MCV was seen in $48(77.4 \%)$ of males and $26(68.4 \%)$ females. Normal MCV was seen in 23 patients in the study group while only three patients had a raised MCV. Mean corpuscular hemoglobin $(\mathrm{MCH})$ was less than normal range in 82 cases in the study group with a mean of $23.3 \pm 5.3 \mathrm{pg}$. A low MCH was seen in $49(79.0 \%)$ of males and 33 (86.8\%) of females. MCH was normal in 14 patients while four patients had a raised $\mathrm{MCH}$. Mean corpuscular haemoglobin concentration (MCHC) was less than normal in 57 patients in the study group with a mean value of $30.8 \pm 2.6 \mathrm{~g} / \mathrm{dl}$. A low MCHC was seen in $31(50 \%)$ males and $26(68.4 \%)$ females. 38 patients had a normal MCHC, while it was increased in five patients. Red cell distribution width (RDW) was increased in 94 patients in the study group with a mean RDW of $18.1 \pm 3.1 \%$. A raised RDW was seen in $57(91.9 \%)$ males and 37 (97.4\%) females. It was normal in the remaining 6 patients. (TABLE 2)

Analysis of PBF revealed a microcytic hypochromic smear in 62 patients, dimorphic smear in 31 patients, normocytic normochromic smear in five patients and macrocytic blood smear in two patients. (TABLE 3)

Amongst the 100 patients in the study group, five patients had an abnormal haemoglobin electrophoresis and 95 had a normal electrophoresis study. Three patients had an abnormal $\mathrm{HbA}_{2}$ band along with $\mathrm{HbA}$ (Beta thalassemia trait) - (Figure 1). One patient had abnormal $\mathrm{HbF}$ and $\mathrm{HbA}$ band (Beta thalassemia 
major) - (Figure 2) and one patient had an abnormal band in the HbSD region - (Figure 3) (HbS confirmed by sickling) - HbS Disease.

\section{Analysis Of Abnormal Electrophoresis Samples}

The mean hemoglobin in patients with beta thalessmia trait was $8.6 \mathrm{~g} / \mathrm{dl}$, mean $\mathrm{RBC}$ count 5.4million/ $\mu$ l, mean MCV 63.5fL, mean MCH 20.7pg, mean MCHC 32.0g/dl, mean RDW $15.2 \%$ with two patients having microcytic hypochromic smear and one patient having dimorphic smear. The patient with beta thalassemia major on electrophoresis had hemoglobin of $6.1 \mathrm{~g} / \mathrm{dl}$, RBC count 2.8 million $/ \mu 1, \mathrm{MCV} 72.0 \mathrm{fL}, \mathrm{MCH}$ 21.3pg, MCHC 29.6g/dl, RDW 26.9\% with microcytic hypochromic blood film. The patient with HbS disease had hemoglobin of $8.6 \mathrm{~g} / \mathrm{dl}$, RBC count 5.5 million/ $\mu 1$, MCV 63.0fL, MCH 21.0pg, MCHC 34.0g/dl, RDW $14.9 \%$ with a dimorphic blood film. (TABLE 4)

Overall the mean age of patients with abnormal haemoglobin pattern was $12.2 \pm 4.9$ years, mean haemoglobin of $8.0 \pm 1.0 \mathrm{~g} / \mathrm{dl}$. Of the patients with abnormal haemoglobin electrophoresis $60 \%$ were males and $40 \%$ were females. The mean RBC count was $4.3 \pm 1.4$ million $/ \mu 1$, MCV was $72.4 \pm 14.1 \mathrm{fl}$, MCH was $22.9 \pm 4.2 \mathrm{pg}$, MCHC was $31.4 \pm 1.5 \mathrm{~g} / \mathrm{dl}$ and RDW was $18.1 \pm 4.6 \%$. In the group of patients with abnormal electrophoretic pattern, $60 \%$ had microcytic hypochromic smear on peripheral blood film and $40 \%$ had dimorphic smear. There was no statistical significant difference of these values in patients with normal versus abnormal hemoglobin electrophoretic pattern. (TABLE 5)

\section{Discussion}

In the $21^{\text {st }}$ century, anemia still remains one of the most common cause of morbidity in India, with prevalence of anemia in children in the age group of 6-35 months to be $73.6 \%{ }^{[5]}$ Hemoglobinopathy and thalassemia have a carrier frequency that ranges from 3 to $17 \%$ in Indian population. The analysis is based upon 83 population groups from 15 states of India for hemoglobin D, 93 populations for hemoglobin E and 308 populations for sickle cell hemoglobin. ${ }^{[1]}$ In a study conducted on sample population of northern India, $12.5 \%$ of the study population was found to be having hemoglobin variants with beta thalassemia trait forming the largest subgroup - to the tune of $8.9 \% .{ }^{[6]}$

It is important to make an accurate and early diagnosis of the type of anemia so as to institute appropriate treatment. RBC indices and examination of the peripheral blood film alone does not exclude majority of hemoglobinopathy traits and thalassemia. ${ }^{[2]}$ Hemoglobin electrophoresis at alkaline $\mathrm{pH}$ is the single most important investigation in preliminary screening of abnormal hemoglobin. Agar gel electrophoresis is simple, rapid and cost effective method of hemoglobin electrophoresis. ${ }^{[3]}$

Our present study showed the prevalence of hemoglobinopathies in the Punjabi population in children with anemia to be $5 \%$. This is comparable to the study by Balgir who found the prevalence range between 3 $17 \%$ in Indian population (in 15 states). ${ }^{[1]}$ However study by Sachdev et al in Northern India showed the prevalence of hemoglobin variants was $12.5 \% .{ }^{[6]}$

The mean values of hemoglobin and $\mathrm{RBC}$ indices of cases of beta thalassemia major in the study by Patel et al in Gujarat is comparable to the present study ${ }^{[7]}$ Shivashankara et al in a study in Karnataka on children with anemia found 15 cases of beta thalassemia major with mean values of $\mathrm{MCV}, \mathrm{MCH}$ that is also comparable to the present study. ${ }^{[8]}$

The prevalence of beta thalassemia trait was $8.9 \%$ in study by Sachdev et al. ${ }^{[6]}$ The study on 11,000 school children of Delhi and Mumbai done by Madan et al showed that the prevalence of beta thalassemia trait in Delhi school children was $5.47 \%$ as against $2.68 \%$ in Mumbai. ${ }^{[9]}$ However in the present study the prevalence rate is $3 \%$. We attribute this fact due to the relative small study population in our study. The hemoglobin value in the present study of patients with beta thalassemia trait was $8.57 \mathrm{~g} / \mathrm{dl}$, as against 10.9 $12.2 \mathrm{~g} / \mathrm{dl}$ in the study by Madan et al. RBC count, MCV, MCH and MCHC is comparable. ${ }^{[9]}$

In the present study we have one case $(1 \%)$ with abnormal electrophoretic band in the $\mathrm{D}, \mathrm{S}$ region which was confirmed to HbS by sickling test. This is low as compared to the average gene frequency of hemoglobin S observed in India (4.3\%) as shown by Balgir. ${ }^{[10]}$ However Sachdev et al in a study of 2600 cases from North India reported only one case with HbS by HPLC $(0.03 \%) .{ }^{[6]}$ Giri et al in a study by agar gel electrophoresis found one out of 51 cases with abnormal hemoglobinopathy to be HbS. ${ }^{[3]}$ The mean hemoglobin value, MCHC and RDW of case of sickle cell disease in the present study is comparable to that of study conducted by Patel et al. ${ }^{[7]}$

The mean hemoglobin and RBC indices values of cases of beta thalassemia trait, beta thalassemia major and sickle cell disease in the present study are also comparable to the values in similar cases of hemoglobinopathies obtained in a study of electrophoretic patterns conducted by Rao et al in 2010 on 800 suspected hemoglobinopathy cases in AIIMS. ${ }^{[1]}$

In a study done in AIIMS by Tyagi et al where HPLC was performed on all cases to check the accuracy of agar gel electrophoresis, they found good correlation between carefully performed conventional methods and 
HPLC-especially in beta homozygous thalassemia, $\mathrm{HbE}$ and $\mathrm{HbS}$ syndromes. They reported advantage of HPLC over conventional electrophoresis only in limited cases and is a must only in cases with hemoglobin migration in SDG region.

\section{Conclusions}

Hemoglobin electrophoresis by agar gel electrophoresis is a cost effective method for screening and diagnosis of patients with suspected hemoglobinopathies as PBF and RBC indices cannot help diagnosis the same; however it is time consuming and requires proper standardization.

\section{References}

[1]. Balgir RS. The burden of haemoglobinopathies in India and the challenges ahead. CurrSci 2000;79:1536-47.

[2]. Davis L.R. Target cells in hemoglobinopathies. J ClinPathol 1972;25:169-70.

[3]. Giri DD, Patra SB, Patel RZ. Hemoglobin electrophoresis in agar gel - A modified method for routine use. Indian J PatholMicrobiol 1984;27:179-83.

[4]. Tyagi S, Saxena R, Choudhry VP. HPLC- How necessary is it for haemoglobinopathy diagnosis in India? Indian J PatholMicrobiol 2003;46:390-3.

[5]. Technical consultation on "Strategies for Prevention and control of Iron Deficiency Anemia amongst under three children in India". Indian Pediatrics 2002;39:640-7.

[6]. Sachdev R, Dam AR, Tyagi G. Detection of Hb variants and hemoglobinopathies in Indian population using HPLC: Report of 2600 cases. Indian J PatholMicrobiol 2010;53:57-62.

[7]. Patel J, Patel A, Patel J, Kaur A, Patel V. Prevalence Of Haemoglobinopathies In Gujarat, India: A Cross-Sectional Study. Int J Hematol 2009;5:1.

[8]. Shivashankara A.R, Jailkhani R, Kini A. Hemoglobinopathies in Dharwad, North Karnataka: A Hospital - Based Study. Journal of Clinical and Diagnostic Research 2008;2:593-9.

[9]. Madan N, Sharma S, Sood SK, Colah R, Bhatia HM. Frequency of $\beta$-thalassemia trait and other hemoglobinopathies in northern and western India. India J of Human genetics 2010;16:16-25.

[10]. Balgir RS. Genetic epidemiology of the three predominant abnormal hemoglobins in India. J Assoc Physicians India 1996;44:25-8.

[11]. Rao S, Kar R, Gupta SK, Chopra A and Saxena R. Spectrum of haemoglobinopathies diagnosed by cation exchange-HPLC \& modulating effects of nutritional deficiency anaemias from north India. Ind J Med Res 2010 Nov;132:513-9.

\section{Legends To Figures}

Figure 1 -Abnormal HbA2 band (Beta Thalassemia Trait)

Figure 2 - Abnormal $\mathrm{HbF}$ and $\mathrm{HbA}$ band (Beta Thalassemia Major)

Figure 3 - Abnormal band in $\mathrm{HbSD}$ region $(\mathrm{Hb} \mathrm{S}$ )

Tables

Table 1 - Age \& Hemoglobin

\begin{tabular}{|c|c|c|c|}
\hline & Mean - Overall & Mean - Males & Mean - Females \\
\hline Age & $7.4 \pm 5.6 \mathrm{yrs}$ & $6.3 \pm 5.2 \mathrm{yrs}$ & $9.1 \pm 5.7 \mathrm{yrs}$ \\
\hline Hemoglobin & $7.4 \pm 1.4 \mathrm{~g} / \mathrm{dl}$ & $7.6 \pm 1.3 \mathrm{~g} / \mathrm{dl}$ & $7.2 \pm 1.6 \mathrm{~g} / \mathrm{dl}$ \\
\hline
\end{tabular}

Table 2 - Red Blood Cell Indices

\begin{tabular}{|c|c|c|c|}
\hline RBC INDICES & Overall & Males & Females \\
\hline RBC Count & $\begin{array}{c}\text { Normal }-10 \text { patients } \\
\text { Low }-82 \text { patients }\end{array}$ & Low $-82.3 \%$ & Low $-81.6 \%$ \\
\hline MCV & $74.0 \pm 13.5 \mathrm{fL}$ & Low $-77.4 \%$ & Low $-68.4 \%$ \\
\hline MCH & $23.3 \pm 5.3 \mathrm{pg}$ & Low $-79.0 \%$ & Low $-86.8 \%$ \\
\hline MCHC & $30.8 \pm 2.6 \mathrm{~g} / \mathrm{dl}$ & Low $-50 \%$ & Low $-68.4 \%$ \\
\hline RDW & $18.1 \pm 3.1 \%$ & High $-91.9 \%$ & High $-97.4 \%$ \\
\hline
\end{tabular}

Table 3 - Peripheral Blood Film

\begin{tabular}{|c|c|c|c|}
\hline PBF & Overall & Males & Females \\
\hline Microcytic Hypochromic & 62 patients & $66.1 \%$ & $55.3 \%$ \\
\hline Dimorphic & 31 patients & $27.4 \%$ & $36.8 \%$ \\
\hline Normocytic normochromic & 5 patients & $6.5 \%$ & $2.6 \%$ \\
\hline Macrocytic & 2 patients & 0 & $5.3 \%$ \\
\hline
\end{tabular}

Table 4 - Abnormal Electrophoresis Parameters

\begin{tabular}{|c|c|c|c|}
\hline Parameters & $\begin{array}{c}\text { Beta Thalassemia } \\
\text { Trait }\end{array}$ & Beta Thalassemia Major & Hbs Disease \\
\hline Numbers & 3 & 1 & 1 \\
\hline $\mathrm{Hb}$ & $8.6 \mathrm{G} / \mathrm{Dl}$ & $6.1 \mathrm{G} / \mathrm{Dl}$ & $8.8 \mathrm{G} / \mathrm{Dl}$ \\
\hline $\begin{array}{c}\text { Rbc Count } \\
\text { Million} / \mu \mathrm{l})\end{array}$ & 5.4 & 2.8 & 5.5 \\
\hline $\mathrm{Mcv}$ & $63.5 \mathrm{fl}$ & $72.0 \mathrm{fl}$ & $63.0 \mathrm{fl}$ \\
\hline
\end{tabular}


Hemoglobin Electrophoretic Patterns In Punjabi Children With Anemia

\begin{tabular}{|c|c|c|c|}
\hline Mch & $20.7 \mathrm{pg}$ & $21.3 \mathrm{pg}$ & $21.0 \mathrm{pg}$ \\
\hline Mchc & $32.0 \mathrm{G} / \mathrm{Dl}$ & $29.6 \mathrm{G} / \mathrm{Dl}$ & $34.0 \mathrm{G} / \mathrm{Dl}$ \\
\hline Rdw & $15.2 \%$ & $26.9 \%$ & $14.9 \%$ \\
\hline Smear & $\begin{array}{c}\text { Microcytic } \\
\text { Hypochromic }-2 \\
\text { Dimorphic }-1\end{array}$ & $\begin{array}{c}\text { Microcytic Hypochormic }- \\
1\end{array}$ & Dimorphic -1 \\
\hline Abnormal Bands & Hba2 & Hbf & Hbs \\
\hline
\end{tabular}

Table 5 - Comparison (Normal And Abnormal Electrophoresis)

\begin{tabular}{|c|c|c|}
\hline Variables & Normal Electrophoresis & Abnormal Electrophoresis \\
\hline Number & 95 & 5 \\
\hline Age & $7.1 \mathrm{Yrs}$ & $12.2 \mathrm{Yrs}$ \\
\hline Hemoglobin & $7.4 \mathrm{G} / \mathrm{Dl}$ & $8.0 \mathrm{G} / \mathrm{Dl}$ \\
\hline Male $:$ Female & $1.6: 1$ & $4.5: 1$ \\
\hline Rbc Count & $3.2 \mathrm{million} / \mu \mathrm{million} / \mu 1$ \\
\hline Mcv & $73.9 \mathrm{Fl}$ & $72.4 \mathrm{Fl}$ \\
\hline Mch & $23.2 \mathrm{Pg}$ & $22.9 \mathrm{Pg}$ \\
\hline Mchc & $30.8 \mathrm{G} / \mathrm{Dl}$ & $31.3 \mathrm{G} . \mathrm{Dl}$ \\
\hline Rdw & $18.1 \%$ & $18.1 \%$ \\
\hline Microcytic Hypochromic & $63.2 \%$ & $60 \%$ \\
\hline Normocytic Normochromic & $5.3 \%$ & $0 \%$ \\
\hline Dimorphic & $30.5 \%$ & $40 \%$ \\
\hline Macrocytic & $2.1 \%$ & $0 \%$ \\
\hline
\end{tabular}

